that act on the cause of a disease, and to license them "ready for the clinic" to pharmaceutical partners. According to Bruno Tocque, ExonHit's chief executive officer, "[d]rugs affect splicing in a cell-typeand drug-specific way. DATAS identifies such drug- and disease-related signatures and can be put to fruition to follow up drug efficacy and patient selection." DATAS technology is therefore being applied to pharmacogenomics-providing a means to identify the best treatment for each patient, while reducing the risk of adverse side effects.

Indeed, ExonH it's first product is a predictive toxicology kit-Safe-Hit. Launched in 1999, Safe-Hit isa DNA array that can detect the splice variants induced by toxic stress on cells, providing a means to determinethe relativetoxicity of compounds. Another two diagnostic tools arein development: Proof-Hit, a diagnostic tool to determinepatients who will respond to standard chemotherapy; and Profile-Hit, a test to screen out patients at risk of severe adverse drug reactions. ExonH it has several products in the pipeline: a research tool for neurodegenerative diseases; three diagnostics including one in clinical trials for cancer therapies, and one for presymptomatic detection of "mad-cow" disease.

\section{ED}

\title{
Signature Bioscience
}

\author{
Catching proteins in action.
}

roteins have two defining proper-

ties: structure and mobility.

Although many companies are cur-

rently elucidating protein structure

using $x$-ray crystallography and nuclear

magnetic resonance (NM R) spectroscopy,

few are studying how proteins and their

domains move during physical interaction

with other molecules. Signature Bioscience has sought to remedy this situation using its technology platform, multipole coupling spectroscopy (MCS).

First conceived by John Hefti, M CS involves probing proteins with microwaves and recording the resulting electromagnetic spectra, creating a unique "signature" for each protein. The protein is then combined with a small molecule, spliceosome, or cell, and a new signature obtained.

Changes in the protein's signature may reflect various characteristics of the protein, including domain movements, enzymatic activity, conformational change, or denaturation. M CS can therefore be used to determine whether a drug candidate binds to a protein target, and can detect cellular alterations such as changes in

Founded: October 1998

Founders: John Hefti (chief technology officer)

CEO: Mark McDade

Employees: 65

Financing to date: $\$ 21$ million

Location: Hayward, CA

http://www.signaturebio.com morphology, ion redistribution and flux, and protein redistribution within a cell.

H efti says that MCS can also determine if two enzymes with similar amino acid sequences have radically different structures and therefore different functions, or whether two enzymes with dissimilar sequence have similar structures and therefore related functions. Such information is valuable when mapping protein-protein interactions, in which a crystallographic approach would be both difficult and timeconsuming.

The technology itself is not original; it is identical to methods used by computer chip engineers to determinethe purity of semiconductor crystals. However, Signature Bioscience has been able to eliminate what had been the main limitation to the technique: the background noise generated by water in the solutions of proteins. A similar problem arises with the noise created by the cell membranes in which most important drug targets are embedded, but H efti is confident that the company will eventually overcome this difficulty.

Signature Bioscience is also developing software tools that can translate the raw data into valuable information. Within two years, revenue will be generated by licensing out a "package" of database and software for protein mapping and drug screening of the 100 protein families that are the most popular drug targets. Some revenue with begenerated through the marketing of spectroscopesmanufactured with a partner-but Signature Bioscience's main profit generator will betarget-validation collaborations. The company currently has three collaborations, including a deal with Sunesis Pharmaceuticals (Redwood City, CA) to determine smallmoleculeinhibitors of protein-protein interactions within inflammation pathways. By the end of theyear, H efti hopes that Signature Bioscience will be able to carry out 1,000 assays per day per machine, further attracting customers to its unique database.

\section{Protein Pathways}

\section{Discovering conservation among molecular targets.}

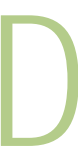

etermining the function of the 100,000 or more proteins that underlieall human biochemical processes is a formidabletask. M ost proteins are assigned a function through studies of their homology with other proteins whose function is known, but this has severe limitations. Protein Pathways is now employing in tandem two approachesphylogenetic profiling and Rosetta stone analysis- that could take the identification of protein function to a new level.

The tools were developed by David Eisenberg at the University of California at Los Angeles (UCLA), who then licensed them exclusively to Protein Pathways.
Phylogenetic profiling is based on the concept that different proteins work together in groups within, for example, a specific

Founded: April 1999

Founders: David Eisenberg, Matteo Pellegrini, Kenneth Goodwill, Michael Thompson, Edward Marcotte, and Todd Yeates

Employees: 14

President: Matteo Pellegrini

Financing to date: $\$ 7$ million

Location: Los Angeles, CA

http://www.proteinpathways.com 